\title{
Level of Understanding of Eras Affects Practitioners' Practice and Perception of Early Postoperative Resumption of Oral Intake: A Nationwide Survey
}

\author{
Huizhen Huang \\ Peking Union Medical College Hospital \\ Yuelun Zhang \\ Peking Union Medical College Hospital \\ Le Shen ( $\square$ pumchshenle@163.com ) \\ Peking Union Medical College Hospital \\ Yuguang Huang \\ Peking Union Medical College Hospital
}

\section{Research Article}

Keywords: Postoperative, Oral intake, Enhanced recovery after surgery, Survey

Posted Date: August 24th, 2021

DOI: https://doi.org/10.21203/rs.3.rs-646185/v1

License: (c) (i) This work is licensed under a Creative Commons Attribution 4.0 International License. Read Full License

Version of Record: A version of this preprint was published at BMC Anesthesiology on November 12th, 2021. See the published version at https://doi.org/10.1186/s12871-021-01500-9. 


\section{Abstract}

Background: Early postoperative resumption of oral intake is supposed to be safe and beneficial to patients recovery. However, practitioners still have great confusion and disagreement about postoperative resumption of oral intake. This is a nationwide survey to investigate the current status of clinical practice and practitioners' attitude toward postoperative resumption of oral intake along with their level of understanding of the ERAS guidelines.

Methods: An anonymous web-based survey questionnaire via mobile social platform was carried out in mainland China from December 11-20, 2020. The Wilcoxon signed rank test or chi-square test was used to compare the propensity of the resumption of oral intake.

Results: Totally 5370 responses were received, and $89 \%$ of them were from anesthesiology departments. The nature of the responses from clinical practitioners was highly diverse, but each of the three surgery types showed unique patterns of ERAS implementation. The respondents were more conserved regarding the commencement of both fluid and solid diets after gastrointestinal (GI) and hepato-pancreato-biliary (HPB) surgery than after non-abdominal (NA) surgery. Most respondents agreed that early oral intake is beneficial to reduce postoperative complications improve bowel recovery and overall outcome. 55\% respondents considered themselves to have a better understanding of ERAS and tended to initiate oral intake early for all three surgery types $(P<0.001)$.

Conclusions: The postoperative resumption of oral intake is highly variable among GI, HPB and NA surgeries. A better understanding of ERAS would encourage practitioners to commence oral intake resumption much earlier.

\section{Background}

Traditionally, postoperative oral intake is gradually introduced following the resumption of bowel sounds and the passage of flatus or stool, which is mainly due to the fear that early oral intake can prolong paralytic ileus. However, a growing body of evidence has suggested these fears are ungrounded, and it has been widely accepted that any delay in the resumption of a normal oral diet after major surgery is associated with increased rates of surgical site infection and delayed recovery $(1-3)$. As it is safe and beneficial to patients, the concept of early postoperative oral intake has been increasingly incorporated into enhanced recovery after surgery (ERAS) protocols $(4,5)$. ERAS clinical pathways have been developed with the goal of improving the quality of perioperative care, minimizing the loss of functional capacity and enhancing the recovery process. The ERAS program initially became well established for colorectal surgery (6) and has been further expanded, promoting rapid functional recovery after abdominal and nonabdominal surgeries such as gastrointestinal surgery(7), liver surgery(8), lung surgery(9), lung surgery(10), breast surgery (11), lumbar spinal fusion(12), lumbar spinal fusion(13), cardiac surgery(14) and gynecological surgeries $(15,16)$. 
Almost all these ERAS programs suggest re-establishing oral feeding as early as possible after surgery. Recently, the European Society for Clinical Nutrition and Metabolism (ESPEN) nutrition guidelines(17) have recommended that in general, oral nutritional intake should be continued after surgery without interruption and that oral intake including clear liquids should be initiated within hours after surgery in most patients. (Evidence level: I, Recommendation grade: A). However, considering the original bowel function state and the impact of surgery is variable among patients with different conditions, the guidelines also noted that oral intake should be adapted according to individual tolerance and to the type of surgery carried out, with special caution taken for elderly patients. It deserves due attention that unlike the "2-4-6-8 rule" as a universally accepted standard of preoperative fasting(18), when to commence postoperative oral intake is vague and confusing.

On the other hand, despite the clinical benefits of early resumption of oral intake, its implementation in clinical practice has been relatively "delayed". Possible reasons include a lack of convincing data, a lowlevel understanding of ERAS, and limitations in institutional experience and systems(19). Therefore, in an effort to identify difficulties in the implementation of early postoperative oral intake programs, we carried out a nationwide survey to determine clinical practices and attitudes toward the postoperative resumption of oral intake among all clinical practitioners along with their level of understanding of ERAS.

\section{Methods}

Ethics: Ethical approval for this study (Ethical Committee No. S-K984) was provided by the Ethical Committee of Peking Union Medical College Hospital, Beijing, China (Chairperson Prof Zhaohui Zhu) on December 11, 2019. As the participation was voluntary and no patients were involved, the requirement for written informed consent was waived.

The "Checklist for Reporting the Results of Internet E-Surveys" (CHERRIES) statement was used for this web-based survey study to improve the quality of reports(20). We composed an anonymous electronic survey using an online survey tool (https://www.wenjuan.com/). The questionnaire of this survey consisted of 18 single choice, multiple choice and five-point Likert scale questions. If necessary, questions were given an additional free-text option for comments. The survey was piloted and further refined based on initial feedback. The survey questions and structure are shown in Appendix 1 . The survey was distributed via WeChat, which is a widely used mobile social platform that attracts a broad range of users in China. Each mobile phone was allowed to submit the questionnaire only once to prevent duplicates. The survey was live from December 11-20, 2020.

We compared the data between different categories of surgeries and between clinical practices and clinician perceptions using the Wilcoxon signed rank test. We compared the data from respondents that had a good understanding of the ERAS with the data from respondents who had less understanding of the ERAS using the chi-square test. All data were analyzed with SPSS [version 20 (SPSS, Chicago, IL, USA)], and two-sided $p$ values $<0.05$ were considered statistically significant. 


\section{Results}

A total of 5370 completed questionnaires were collected, the average response time was 20 minutes, and the completion rate was $40.71 \%$. The major characteristics of the respondents are shown in Fig. 1. Most of the respondents were from anesthesiology departments (89\%), followed by surgical departments (8\%) and nonsurgical departments (3\%). Eighty-five percent of the respondents were doctors, including 3621 attendings and 944 residents. Nurses were also invited to complete this survey, accounting for $15 \%$ of all responses. The respondents were asked to take a self-assessment on their degree of understanding of ERAS in the form of a question based on a five-point Likert scale (know well/know some/know a little/know little/do not know). Most claimed that they "know some" (41\%), while 7\% reported "do not know". Based on their understanding of ERAS, respondents were subdivided into two groups: better understanding of ERAS group (know well \& know some) $(n=2954,55 \%)$ and less understanding of ERAS group (know a little, know little and do not know) $(n=2416,45 \%)$ (Fig. 1C).

\section{Postoperative oral intake in practice and perception}

Figure 2 shows an overview of the commencement of postoperative oral intake. The nature of the responses from the clinical practitioners was highly diverse, while each of three surgeries showed unique patterns. Respondents took a more conserved approach for gastrointestinal and hepato-pancreato-biliary surgery, in which almost half (at least 47\%) allowed patients to begin oral intake "6 hours postoperation" "upon removal of nasogastric tube" or "until passage of flatus". While compared to the other two surgeries, non-abdominal surgery showed more concentrated patterns in the resumption of oral liquids and solid diet. For non-abdominal surgery, most physicians allowed oral fluids after "discharge from postanesthesia care unit (PACU)" or just "2-4 hours post operation". Regardless of which surgery was performed, respondents asked patients to refrain from eating longer than drinking. Moreover, although the majority of responses regarding the postoperative resumption of oral fluids in gastrointestinal and hepato-pancreato-biliary surgery were not fixed, the respondents showed a surprising amount of consensus in regard to the resumption of a solid diet-namely, "after the passage of flatus" (in each column, $>38 \%$ in the former group and $>30 \%$ in the latter group).

When combined with the horizontal axis-understanding of ERAS-the color strips and numbers (representing the percentage of respondents in each group) clearly indicate that respondents that consider themselves to have a better understanding of ERAS tended to commence oral intake early. A close look at the bottom half of figures revealed that clinical practitioners who considered themselves more familiar with ERAS were more likely to commence oral intake once discharged from the PACU or "24 hours postoperation", rather than "6 hours postoperation". On the other hand, respondents who considered themselves less familiar with ERAS ("do not know") reported more difficulty in deciding when to commence of postoperative oral intake ("not sure"), regardless of whether oral fluids or a solid diet was being considered. 
Figure 3 shows an overview of the postoperative resumption of oral intake in terms of respondent expectations. The patterns between clinical practice and perception were comparable, and similar to practice, there was a strong tendency for respondents who knew less about ERAS to appear more conserved in their expectations. Overall though, respondents' expectations were more aggressive than their practice. It is very interesting that clinicians who responded that they "know well" ERAS had the minimum relative difference between practice and perception, exhibiting the most confidence in their decision regarding early resumption of oral intake. Among all the options, the fewest number of respondents waited to resume postoperative oral intake "until the passage of feces" in both practice and perception, regardless of surgery type.

\section{Surgical and perception differences in the early postoperative resumption of oral intake}

This study focused on early oral intake; thus, for clear analysis, early resumption of postoperative oral intake was defined as diet given no more than 6 hours postoperatively to patients, including the timepoints of "discharge from PACU" and "2-4 hours postoperation", while late postoperative oral intake included all other timepoints except those who chose "not sure". As shown in Fig. 4, despite the pattern similarity between gastrointestinal and hepato-pancreato-biliary surgery types, due to the sufficient number of responses, we found significant differences between each surgery group $(P<0.001)$. Regardless of practice or expectation, clinicians seemed to be more conserved regarding the resumption of oral intake after hepato-pancreato-biliary surgery, which may be a reflection of the complexity and heterogeneity of this large group of procedures, including pancreaticoduodenectomy. Obviously, the respondents were more receptive of early oral intake after non-abdominal surgeries. We also noticed that the practice and perception of respondents showed significant differences for each surgery group $(\mathrm{P}<$ 0.001). It is noteworthy that clinicians had more positive perceptions of early postoperative oral intake than reported in terms of practice in all three surgery types. After all, the surgical specialty objectively determined the resumption of oral intake, and the perceptions of the respondents also had an effect.

\section{Attitudes toward the early postoperative resumption of oral intake}

As shown in Fig. 5, for all the listed ERAS components, practitioners with higher level of understanding of ERAS were more likely to agree with the guidelines. Regardless of the level of understanding of ERAS, more than fifty-five percent of the respondents agreed that early postoperative oral intake improves patient satisfaction and overall prognosis and speeds up bowel recovery. However, approximately onethird of practitioners believed that early oral intake after surgery had no effect on reducing postoperative pain. Meanwhile, respondents were more concerned that early oral intake was unfavorable for the alleviation of postoperative nausea and vomiting compared to other ERAS components, even those who claimed they "know well" the benefits of ERAS. As the level of understanding of ERAS increased, respondents reported more confidence in these benefits and less concerned in general. 


\section{Understanding of ERAS affects the postoperative resumption of oral intake}

As mentioned above, the respondents were divided into two groups: better understanding of ERAS and less understanding of ERAS. The differences in respondent characteristics are shown in Table 1. The group with a better understanding had more respondents from the Department of Anesthesiology, consisting of more males and attending physicians. Even though the majority of respondents had a university degree, more respondents in the better understanding group had an advanced degree. All of above differences were significant $(P<0.001)$. 
Table 1

Characteristics of the respondents stratified by their understanding of ERAS

Better Understanding of ERAS ( $\mathrm{n}$ $=2954$ )

= 2416)

$P$ values

Department

Anesthesiology

2726(92)

2054(85)

$<$

$0.001^{*}$

Surgical

180(6)

257(11)

Department

Non-surgical

48(2)

105(4)

Department

Sex

Male

1740(59)

1034(43)

$<$

$0.001^{*}$

Female

1214(41)

1382(57)

Education

College Degree

144(5)

318(13)

$<$

0.001 *

University Degree

1792(61)

1755(73)

Advanced Degree

1018(34)

343(14)

Title

Attending

2321(79)

1300(54)

$<$

0.001 *

Resident

399(14)

545(23)

Nurse

228(8)

$555(23)$

Other

$6(0)$

16(1)

Data presented as $\mathrm{n}(\%) .{ }^{*}$ Chi-square test

Figure 6 shows how the understanding of ERAS affects decisions regarding the postoperative resumption of oral intake. In gastrointestinal surgery, thirty percent of respondents in the better understanding group considered 6 hours after surgery to be a sufficient period for a liquid diet, even though the less understanding group (18\%) preferred conserved treatment. For hepato-pancreato-biliary surgery, we found more conserved answers in both groups, and the difference between the respondents in the better understanding group and those in the less understanding group was slightly larger in terms of early resumption of oral intake. However, after non-abdominal surgery, respondents in the two groups had the largest disagreement on the resumption of oral intake, with $67 \%$ percent of the respondents in the better 
understanding group preferring to resume oral fluid intake early versus only $50 \%$ of the respondents in the less understanding group. For the surgery groups, the pattern of early resumption of oral intake (blue blocks) looks like an upside-down ladder, from fluid to solid diet and from better to less understanding of ERAS, which indicates that a better understanding of ERAS leads to a more favorable choice for resuming oral intake early.

\section{Discussion}

As this nationwide survey aimed to investigate the practice and attitudes towards early postoperative resumption of oral intake along with the understanding of ERAS, it was conducted to collect baseline data that would help establish a proper strategy for the widespread implementation of early postoperative oral intake in clinical practice. We found that early resumption of oral intake after nonabdominal surgery has been relatively well adopted. However, the implementation and conception of ERAS after gastrointestinal and hepato-pancreato-biliary surgery are discrepant, and the approach to recovery for these two types remains relatively conserved. In addition, a better understanding of ERAS would encourage practitioners to commence oral intake earlier in these patients. Therefore, consideration should be given to feasible solutions to the obstacles that may undermine the implementation of ERAS. Finally, a comprehensive and surgery-specific care protocol is urgently required to reduce variation and improve postoperative outcomes in China.

Postoperative resumption of oral intake is highly variable among gastrointestinal, hepato-pancreatobiliary and non-abdominal surgery. Indeed, after abdominal and intestinal surgery, due to the disruption of normal bowel motility, postoperative ileus may occur. For this reason, the timing of postoperative oral intake has long been debated $(21,22)$. Traditional management usually includes remaining fasting until bowel sound or passage of flatus or stool, which are clinical parameters used to confirm resolution of ileus, and then commencing on clear fluids typically 2 to 5 days postoperation and finally progressing to a solid diet as tolerated(23). However, these old-school parameters are dispensable evidence of resumed bowel function(24) and an early oral diet has been shown to be safe at 4 hours after surgery in patients with nondiverted colorectal anastomosis(25). It seems that gastrointestinal surgery is associated with a higher risk of postoperative ileus, so multimodal preventive strategies should be adopted to facilitate the recovery of gastrointestinal function if delayed gastric emptying or ileus occurs after surgery, including the use of nasogastric tubes prophylactically and remedially(7). Moreover, different surgical methods may have different levels of risk, such as gastrointestinal surgery. Esophagojejunostomy is probably a more vulnerable reconstruction than distal or subtotal gastric resection, so patients undergoing the former may need a longer period of postoperative fasting(26). Therefore, for gastrointestinal $(6,7,27,28)$ and hepato-pancreato-biliary $(8,9)$ surgeries, almost all ERAS programs recommend that patients should be allowed to resume a normal diet (may through enteric feeding tubes if needed(28))after surgery without restrictions according to their tolerance, even though none of them mention a specific number of hours or days postoperatively. To further explore early intake after surgery, more large-scale high-quality studies are needed. Our results confirm this clinical need. 
After non-abdominal surgery, clinical practitioners had closer responses regarding the perception and practice of the resumption of oral intake, showing the best implementation and most confidence in ERAS. Indeed, for non-abdominal surgeries, most ERAS guidelines recommended that patients should be encouraged to resume oral liquids and a solid diet as soon as possible $(10,12-14)$, preferably within 24 hours after surgery(11). However, it`s worth noting that most literature cited by above guidelines were retrospective studies or data from intestinal surgery. This arises for a simple reason-namely, that the return to normal food intake is essential component to resuming to normal activities, even though no randomized controlled trials have investigated the direct association of early oral intake with ERAS in these surgery types.

Interestingly, most clinicians had more positive perceptions of ERAS than reported in terms of practice. We found that $4 \%$ of respondents considered that early oral intake after hepato-pancreato-biliary surgery is appropriate, although in practice, they were not sure of the most suitable course of action. Besides, most respondents agreed with the benefits of early oral intake; however, they still chose a conserved strategy in practice. More than half postponed the resumption of oral take due to concerns about choking cough (54\%), postoperative nausea and vomiting (67\%) and aspiration (73\%). In thirty percentage of free text comments, respondents stated that they were concerned of a potential return to the operating room, as fed patients are at risk for aspiration. However, modern anesthetic techniques should mitigate the risk of aspiration in this uncommon scenario(29). While a Cochrane systematic review(30) of colorectal surgery published in 2019 suggests that there is sufficient evidence to indicate that early enteral intake leads to a reduced postoperative length of hospital stay and risk of dying, all referred studies were of low quality. A lack of convincing data is the first stumbling block to ERAS implementation. However, the reality that scientific study does not always allow direct clinical correlation also cannot be ignored.

Among the three kinds of surgeries, respondents with a better understanding of ERAS guidelines were more likely to commence early oral intake after surgery. However, the numbers were not promising; our survey found that only $36 \%$ of respondents stated that they chose the timing of postoperative oral intake based on guidelines, while $55 \%$ chose the timing of postoperative oral intake based on clinical experience. This may be the reflection of the low consensus on the risk and significance of early postoperative oral intake. The current level of implementation of early oral intake in the hospital context is still low and based on type of surgery performed, the physician-patient relationship, and adherence to treatment(31). Indeed, many other factors may influence clinical complication endpoints, such as the fitness of the patient, experience of the surgeon, resection sites, pain control, and success of the operation in resolving the underlying pathology $(32,33)$.

Reestablishment of oral intake as soon as possible after surgery has been incorporated into an increasing number of ERAS programs $(4,5)$. The concept of ERAS involves a multidisciplinary team approach to solve the problems that cause complications and delay recovery by implementing evidencebased care protocols and changes in management through interactive and ongoing audits(34). Our survey found that $22 \%$ practitioners considered that the timing of resumption of oral intake should be only decided and supervised by surgeons and nurses in the wards. But teamwork means engagement of 
all relevant stakeholders. Surgeons should break down entrenched surgical dogmas, and the collaboration of ward nurses is equally important for driving this program forward $(35,36)$. As an anesthesiologist, our analgesic techniques should aim not only to provide optimal pain control but also to facilitate the tolerance of oral intake $(37,38)$ and to prevent postoperative nausea and vomiting using multimodal approaches $(39,40)$. Nonetheless, since early postoperative intake does provide benefit, the identification and implementation of strategies to improve uptake of this ERAS component should be a priority.

As a first nationwide survey focused on the practice and attitudes towards early postoperative resumption of oral intake, there were also some limitations. First, this is a survey designed and conducted by anesthesiologists. Although respondents consisted of practitioners from anesthesiology, surgery and non-surgical departments, $89 \%$ of them are anesthesia related. Considering the feasibility of the study, we did not employ a strict probability sampling method during the study design, which may cause a population bias. Second, the survey or the questions were designed upon current clinical practice of mainland China, so the choices may not reflect current worldwide status of each question. Third, we did not collect the information about practitioners' affiliations, nor did we differ or exclude the respondents who are majored in ambulatory surgery. Finally, all results were from perioperative health care practitioners' answers, but not from the patients. As is known that patients may delayed their resumption of postoperative oral intake despite physicians' arrangements.

\section{Conclusions}

Different from preoperative fasting, practitioners still have great confusion and disagreement about postoperative resumption of oral intake. This study found out that the type of surgery is the key consideration that affects the practitioners' decision to postoperative resumption of oral intake. Postoperative resumption of oral intake is highly variable among surgeries. Regardless of whether it is an abdominal surgery, the practitioners have a relatively consistent view on postoperative resumption of oral intake for the same type of surgery. Clinical practitioners had the best implementation and most confidence in early oral intake after non-abdominal surgery. This suggests that in the ERAS guidelines or consensus for different types of surgery, a clear schedule for postoperative resumption of oral intake could be given for patients without severe complications. In addition, A better understanding of ERAS would encourage practitioners to commence earlier oral intake. Future work should work on enhancing ERAS comprehension, formulating national or regional ERAS protocols and improving the implementation of ERAS, as will promote the overall postoperative recovery.

\section{Declarations}

(1) Ethics approval and consent to participate

The survey was under expedited review and approved by the Institutional Review Board of Peking Union Medical College Hospital on December 11, 2019 (No. S-K984). The IRB file was uploaded this time. As 
this anonymous survey is a minimal risk study, the requirement for written informed consent was waived. The relevant consent information was provided as an introductory screen display before the questionnaire. If the respondent completed the survey, he/she must have agreed to participate.

(2) Consent for publication

I declare to consent for publication and there's no individual identifying data.

(3) Availability of data and material

The datasets generated and analyzed during the current study are available from the corresponding author on reasonable request.

(4) Competing interests

There are no competing interests in this manuscript.

(5) Funding

This work was supported by Education Reform Project Foundation for the Central Universities of Peking Union Medical College (2020zlgc0105), Training Programme Foundation for Excellent Talents in Dongcheng District of Beijing (2019DCT-M-08) and Non-profit Central Research Institute Fund of Chinese Academy of Medical Sciences (2019XK320018). These funding sources had no role in the design of this study, execution, analyses, interpretation of the data, or decision to submit results.

(6) Authors' contributions

$\mathrm{HH}$ designed the survey, collected the data, conduct statistic analysis and drafted the manuscript. $\mathrm{YZ}$ participated in the statistical analysis. LS directed the design of the survey and the study, modify the article. YH conceived of the study, and participated in its design and coordination.

(7) Acknowledgements

We would like to thank all the clinical practitioners who took the time to participate in this research.

\section{References}

1. Andersen HK, Lewis SJ, Thomas S. Early enteral nutrition within $24 \mathrm{~h}$ of colorectal surgery versus later commencement of feeding for postoperative complications. Cochrane Database Syst Rev. 2006(4):CD004080.

2. Lassen K, Kjaeve J, Fetveit T, Trano G, Sigurdsson HK, Horn A, et al. Allowing normal food at will after major upper gastrointestinal surgery does not increase morbidity: a randomized multicenter trial. Ann Surg. 2008;247(5):721-9. 
3. Gerritsen A, Wennink RA, Besselink MG, van Santvoort HC, Tseng DS, Steenhagen E, et al. Early oral feeding after pancreatoduodenectomy enhances recovery without increasing morbidity. HPB (Oxford). 2014;16(7):656-64.

4. Lambert E, Carey S. Practice Guideline Recommendations on Perioperative Fasting: A Systematic Review. JPEN J Parenter Enteral Nutr. 2016;40(8):1158-65.

5. Ljungqvist O, Scott M, Fearon KC. Enhanced Recovery After Surgery: A Review. JAMA Surg. 2017;152(3):292-8.

6. Gustafsson UO, Scott MJ, Hubner M, Nygren J, Demartines N, Francis N, et al. Guidelines for Perioperative Care in Elective Colorectal Surgery: Enhanced Recovery After Surgery (ERAS((R))) Society Recommendations: 2018. World J Surg. 2019;43(3):659-95.

7. Feldheiser A, Aziz O, Baldini G, Cox BP, Fearon KC, Feldman LS, et al. Enhanced Recovery After Surgery (ERAS) for gastrointestinal surgery, part 2: consensus statement for anaesthesia practice. Acta Anaesthesiol Scand. 2016;60(3):289-334.

8. Melloul E, Lassen K, Roulin D, Grass F, Perinel J, Adham M, et al. Guidelines for Perioperative Care for Pancreatoduodenectomy: Enhanced Recovery After Surgery (ERAS) Recommendations 2019. World J Surg. 2020;44(7):2056-84.

9. Melloul E, Hubner M, Scott M, Snowden C, Prentis J, Dejong CH, et al. Guidelines for Perioperative Care for Liver Surgery: Enhanced Recovery After Surgery (ERAS) Society Recommendations. World J Surg. 2016;40(10):2425-40.

10. Batchelor TJP, Rasburn NJ, Abdelnour-Berchtold E, Brunelli A, Cerfolio RJ, Gonzalez M, et al. Guidelines for enhanced recovery after lung surgery: recommendations of the Enhanced Recovery After Surgery (ERAS(R)) Society and the European Society of Thoracic Surgeons (ESTS). Eur J Cardiothorac Surg. 2019;55(1):91-115.

11. Temple-Oberle C, Shea-Budgell MA, Tan M, Semple JL, Schrag C, Barreto M, et al. Consensus Review of Optimal Perioperative Care in Breast Reconstruction: Enhanced Recovery after Surgery (ERAS) Society Recommendations. Plast Reconstr Surg. 2017;139(5):1056e-71e.

12. Wainwright TW, Gill M, McDonald DA, Middleton RG, Reed M, Sahota $\mathrm{O}$, et al. Consensus statement for perioperative care in total hip replacement and total knee replacement surgery: Enhanced Recovery After Surgery (ERAS((R))) Society recommendations. Acta Orthop. 2020;91(1):3-19.

13. Debono B, Wainwright TW, Wang MY, Sigmundsson FG, Yang MMH, Smid-Nanninga $H$, et al. Consensus statement for perioperative care in lumbar spinal fusion: Enhanced Recovery After Surgery (ERAS(R)) Society recommendations. Spine J. 2021.

14. Engelman DT, Ben Ali W, Williams JB, Perrault LP, Reddy VS, Arora RC, et al. Guidelines for Perioperative Care in Cardiac Surgery: Enhanced Recovery After Surgery Society Recommendations. JAMA Surg. 2019;154(8):755-66.

15. Nelson G, Bakkum-Gamez J, Kalogera E, Glaser G, Altman A, Meyer LA, et al. Guidelines for perioperative care in gynecologic/oncology: Enhanced Recovery After Surgery (ERAS) Society recommendations-2019 update. Int J Gynecol Cancer. 2019;29(4):651-68. 
16. Hubner M, Kusamura S, Villeneuve L, Al-Niaimi A, Alyami M, Balonov K, et al. Guidelines for Perioperative Care in Cytoreductive Surgery (CRS) with or without hyperthermic IntraPEritoneal chemotherapy (HIPEC): Enhanced Recovery After Surgery (ERAS(R)) Society Recommendations Part II: Postoperative management and special considerations. Eur J Surg Oncol. 2020;46(12):231123.

17. Weimann A, Braga M, Carli F, Higashiguchi T, Hubner M, Klek S, et al. ESPEN guideline: Clinical nutrition in surgery. Clin Nutr. 2017;36(3):623-50.

18. Fawcett WJ, Thomas M. Pre-operative fasting in adults and children: clinical practice and guidelines. Anaesthesia. 2019;74(1):83-8.

19. Ore AS, Shear MA, Liu FW, Dalrymple JL, Awtrey CS, Garrett L, et al. Adoption of enhanced recovery after laparotomy in gynecologic oncology. Int J Gynecol Cancer. 2020;30(1):122-7.

20. Eysenbach G. Improving the quality of Web surveys: the Checklist for Reporting Results of Internet ESurveys (CHERRIES). J Med Internet Res. 2004;6(3):e34.

21. Harnsberger CR, Maykel JA, Alavi K. Postoperative lleus. Clin Colon Rectal Surg. 2019;32(3):166-70.

22. Lassen $K$, Revhaug A. Early oral nutrition after major upper gastrointestinal surgery: why not? Curr Opin Clin Nutr Metab Care. 2006;9(5):613-7.

23. Bragg D, El-Sharkawy AM, Psaltis E, Maxwell-Armstrong CA, Lobo DN. Postoperative ileus: Recent developments in pathophysiology and management. Clin Nutr. 2015;34(3):367-76.

24. Read TE, Brozovich M, Andujar JE, Ricciardi R, Caushaj PF. Bowel Sounds Are Not Associated With Flatus, Bowel Movement, or Tolerance of Oral Intake in Patients After Major Abdominal Surgery. Dis Colon Rectum. 2017;60(6):608-13.

25. Gustafsson UO, Hausel J, Thorell A, Ljungqvist O, Soop M, Nygren J, et al. Adherence to the enhanced recovery after surgery protocol and outcomes after colorectal cancer surgery. Arch Surg. 2011;146(5):571-7.

26. Warren J, Bhalla V, Cresci G. Postoperative diet advancement: surgical dogma vs evidence-based medicine. Nutr Clin Pract. 2011;26(2):115-25.

27. Mortensen K, Nilsson M, Slim K, Schafer M, Mariette C, Braga M, et al. Consensus guidelines for enhanced recovery after gastrectomy: Enhanced Recovery After Surgery (ERAS(R)) Society recommendations. Br J Surg. 2014;101(10):1209-29.

28. Low DE, Allum W, De Manzoni G, Ferri L, Immanuel A, Kuppusamy M, et al. Guidelines for Perioperative Care in Esophagectomy: Enhanced Recovery After Surgery (ERAS((R))) Society Recommendations. World J Surg. 2019;43(2):299-330.

29. Zdravkovic M, Berger-Estilita J, Sorbello M, Hagberg CA. An international survey about rapid sequence intubation of 10,003 anaesthetists and 16 airway experts. Anaesthesia. 2020;75(3):31322.

30. Herbert G, Perry R, Andersen HK, Atkinson C, Penfold C, Lewis SJ, et al. Early enteral nutrition within 24 hours of lower gastrointestinal surgery versus later commencement for length of hospital stay and postoperative complications. Cochrane Database Syst Rev. 2019;7:CD004080. 
31. Weijs TJ, Berkelmans GH, Nieuwenhuijzen GA, Dolmans AC, Kouwenhoven EA, Rosman C, et al. Immediate Postoperative Oral Nutrition Following Esophagectomy: A Multicenter Clinical Trial. Ann Thorac Surg. 2016;102(4):1141-8.

32. Fujii T, Yamada S, Murotani K, Okamura Y, Ishigure K, Kanda M, et al. Oral Food Intake Versus Fasting on Postoperative Pancreatic Fistula After Distal Pancreatectomy: A Multi-Institutional Randomized Controlled Trial. Medicine (Baltimore). 2015;94(52):e2398.

33. Kuwabara K, Matsuda S, Ishikawa KB, Horiguchi H, Fujimori K. Association of operating time and gastrectomy with initiation of postoperative oral food intake. Dig Surg. 2011;28(3):157-62.

34. Kahokehr A, Sammour T, Zargar-Shoshtari K, Thompson L, Hill AG. Implementation of ERAS and how to overcome the barriers. Int J Surg. 2009;7(1):16-9.

35. Smith TW, Jr., Wang X, Singer MA, Godellas CV, Vaince FT. Enhanced recovery after surgery: A clinical review of implementation across multiple surgical subspecialties. Am J Surg. 2020;219(3):530-4.

36. Brown D, Xhaja A. Nursing Perspectives on Enhanced Recovery After Surgery. Surg Clin North Am. 2018;98(6):1211-21.

37. Guay J, Nishimori M, Kopp SL. Epidural Local Anesthetics Versus Opioid-Based Analgesic Regimens for Postoperative Gastrointestinal Paralysis, Vomiting, and Pain After Abdominal Surgery: A Cochrane Review. Anesth Analg. 2016;123(6):1591-602.

38. Weibel S, Jelting Y, Pace NL, Helf A, Eberhart LH, Hahnenkamp K, et al. Continuous intravenous perioperative lidocaine infusion for postoperative pain and recovery in adults. Cochrane Database Syst Rev. 2018;6:CD009642.

39. Apfel CC, Heidrich FM, Jukar-Rao S, Jalota L, Hornuss C, Whelan RP, et al. Evidence-based analysis of risk factors for postoperative nausea and vomiting. Br J Anaesth. 2012;109(5):742-53.

40. Gan TJ, Diemunsch P, Habib AS, Kovac A, Kranke P, Meyer TA, et al. Consensus guidelines for the management of postoperative nausea and vomiting. Anesth Analg. 2014;118(1):85-113.

\section{Figures}


A

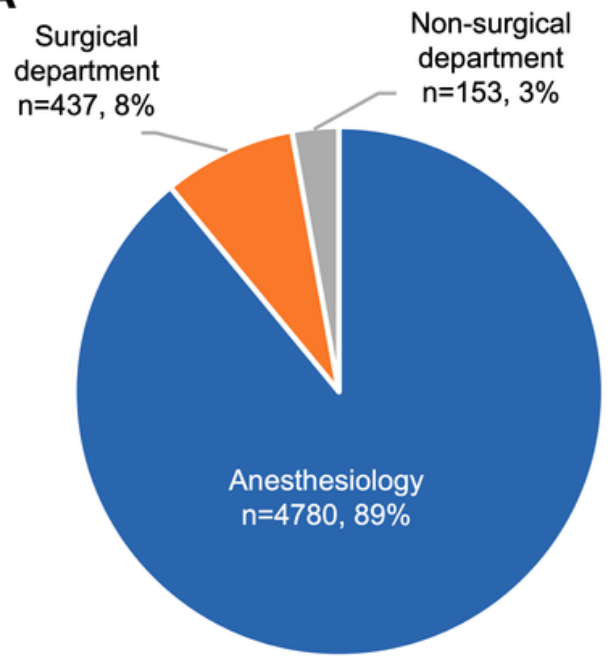

B

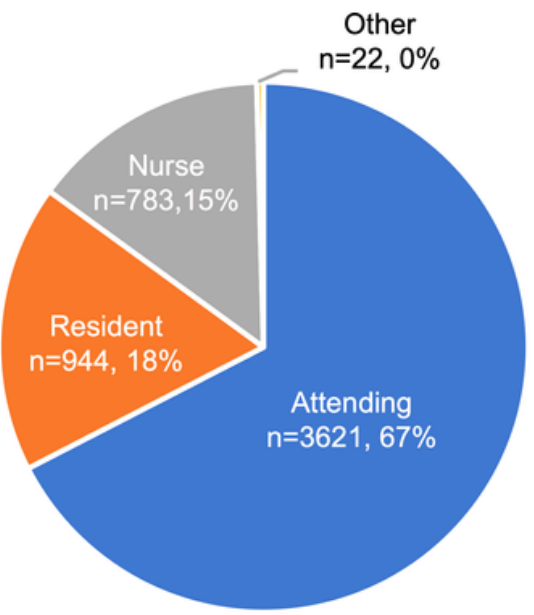

C

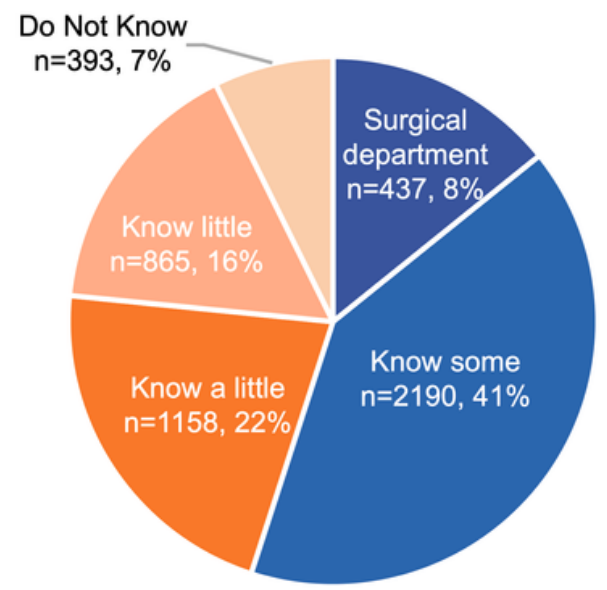

Figure 1

Respondent characteristics, including departments (A), titles (B) and level of understanding about ERAS (C). 
A

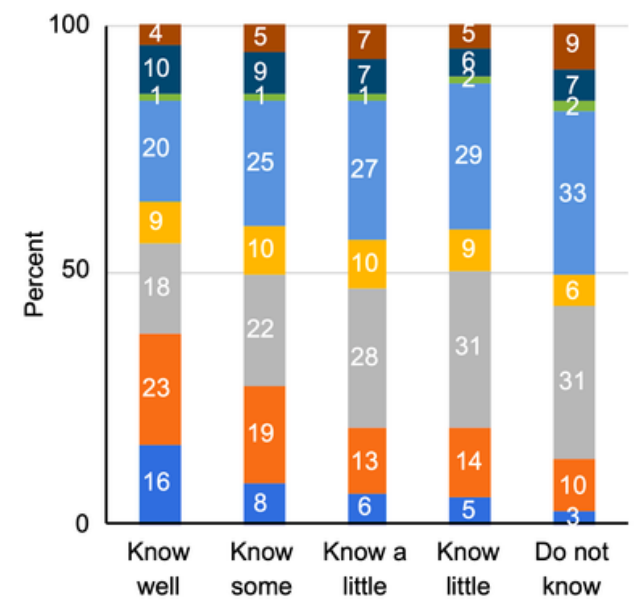

D

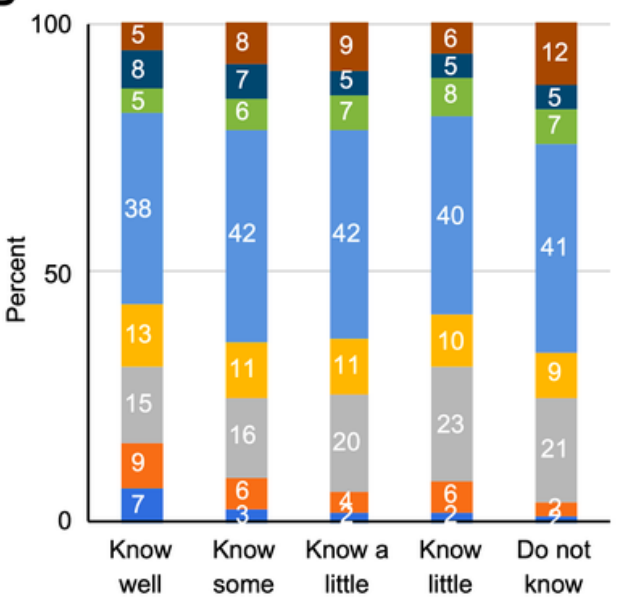

B

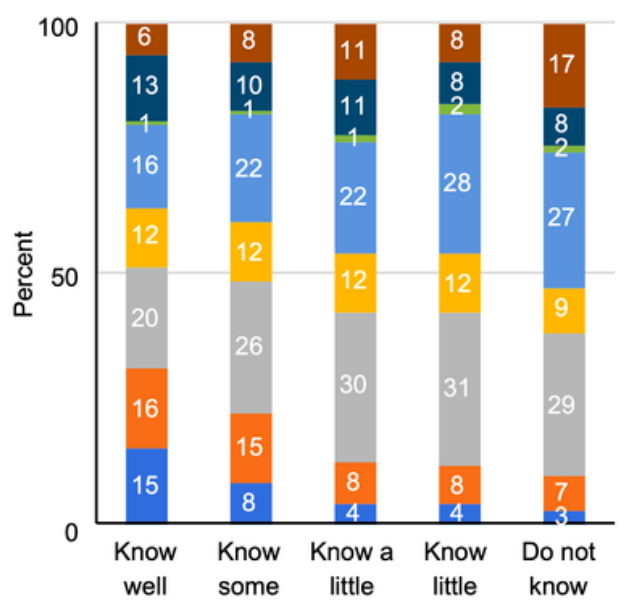

$\mathbf{E}$

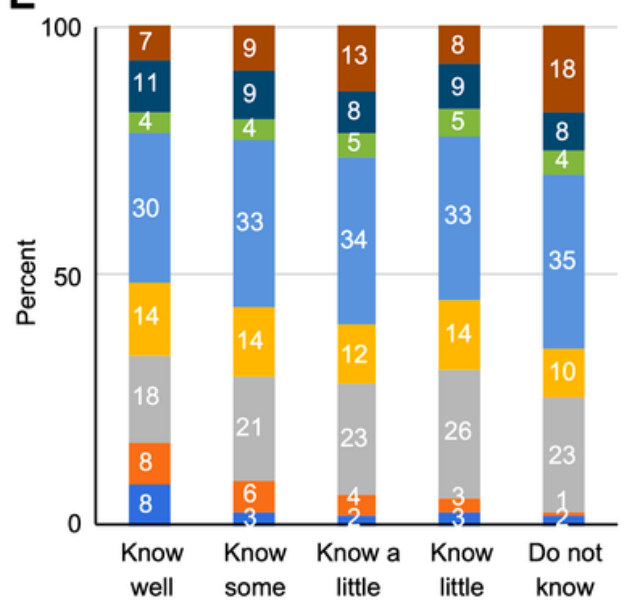

C

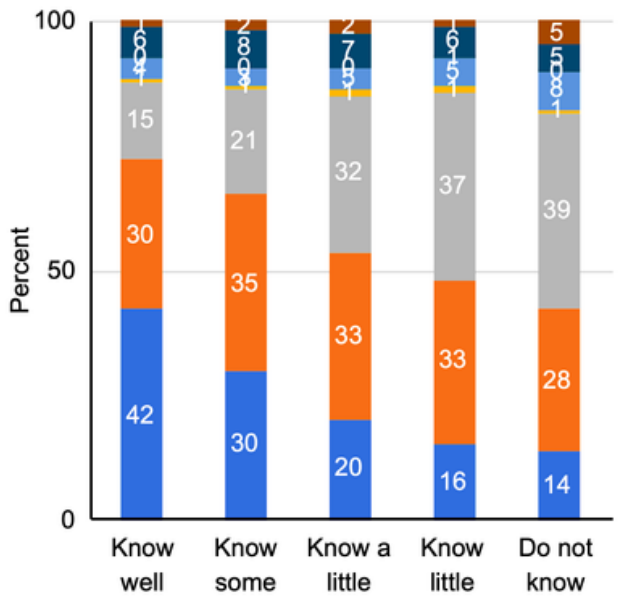

$\mathbf{F}$

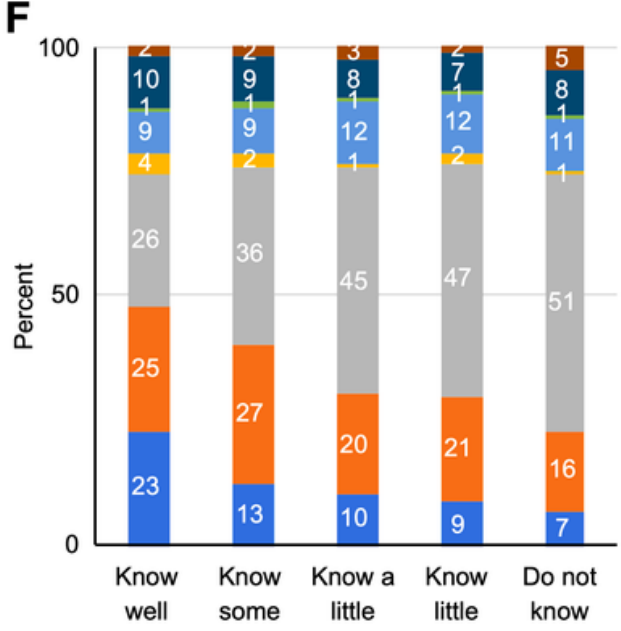

- Discharge from PACU

- 2-4 h post operation $\quad$ a $6 \mathrm{~h}$ post operation

- Upon removal of nasogastric tube = Until passage of flatus = Until passage of feces

- Without nausea, vomiting \&

distension

Figure 2

Overall responses for the postoperative resumption of oral intake in clinical practice along with respondents' level of understanding of ERAS. Resumption of oral fluids after (A) gastrointestinal surgery, (B) hepato-pancreato-biliary surgery, and (C) non-abdominal surgery, and resumption of solid diet after (D) gastrointestinal surgery, $(E)$ hepato-pancreato-biliary surgery, and $(F)$ non-abdominal surgery. PACU, postanesthesia care unit. Data presented as \% in columns. 
A

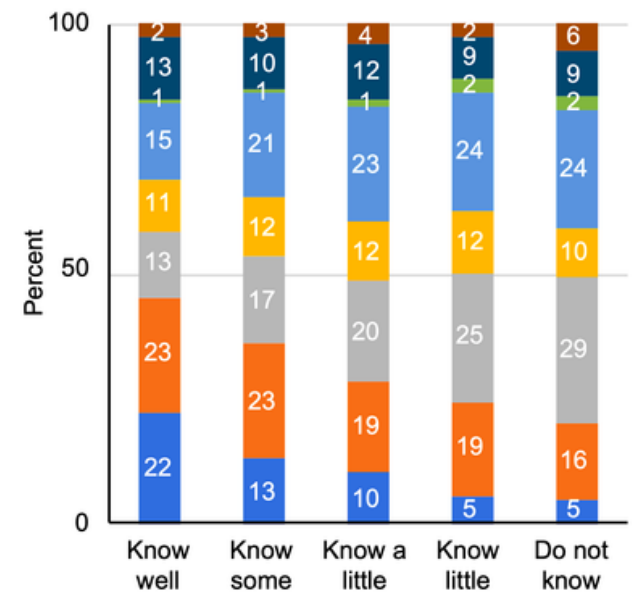

D

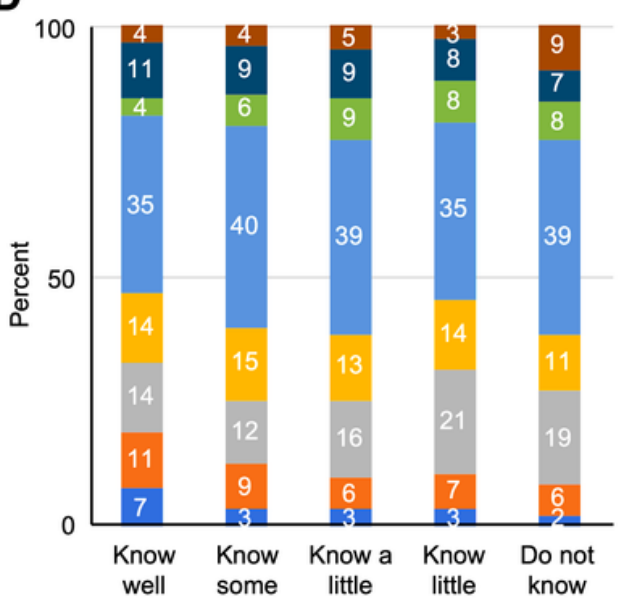

B

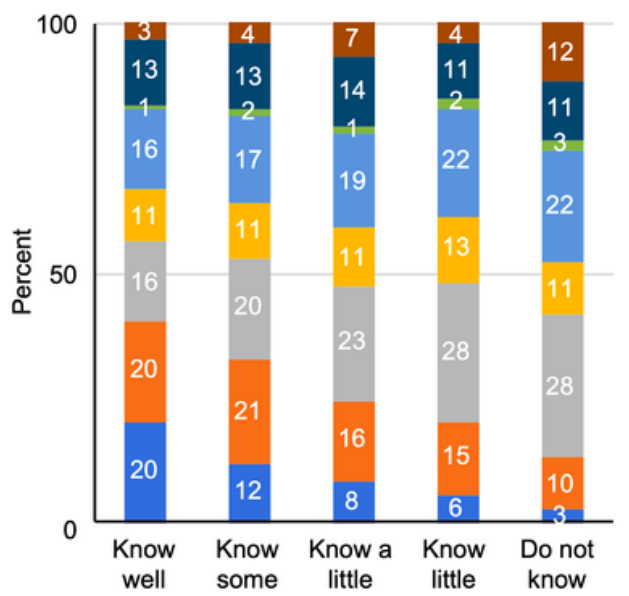

$\mathbf{E}$

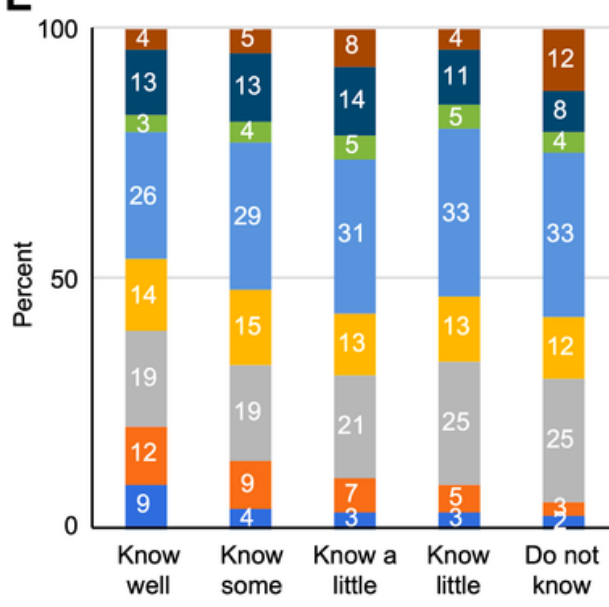

C

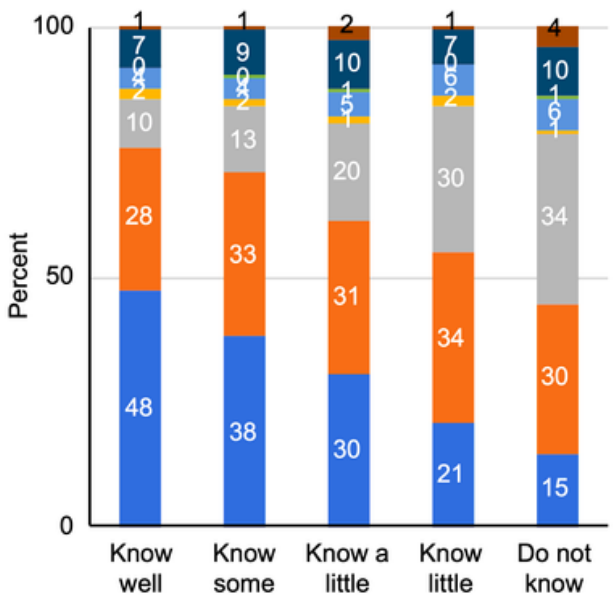

$\mathbf{F}$

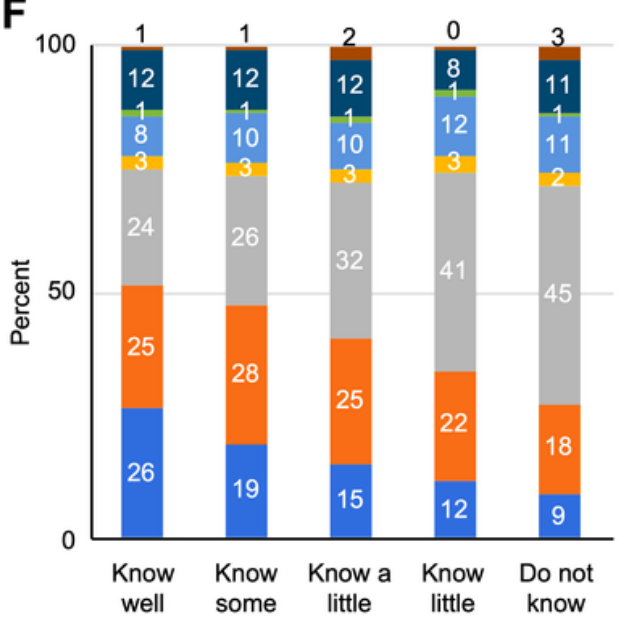

$$
\begin{aligned}
& \text { - Discharge from PACU a 2-4 h post operation } 6 \mathrm{~h} \text { post operation } \\
& \text { - Upon removal of nasogastric tube Until passage of flatus = Until passage of feces } \\
& \text { - Without nausea, vomiting \& } \quad \text { Not sure } \\
& \text { distension }
\end{aligned}
$$

\section{Figure 3}

Overall perception of the postoperative resumption of oral intake along with the respondents' understanding of ERAS. Resumption of oral fluids in (A) gastrointestinal surgery, (B) hepato-pancreatobiliary surgery, and (C) non-abdominal surgery and solid diet in (D) gastrointestinal surgery, (E) hepatopancreato-biliary surgery, and $(F)$ non-abdominal surgery. PACU, postanesthesia care unit. Data presented as $\%$ in columns. 

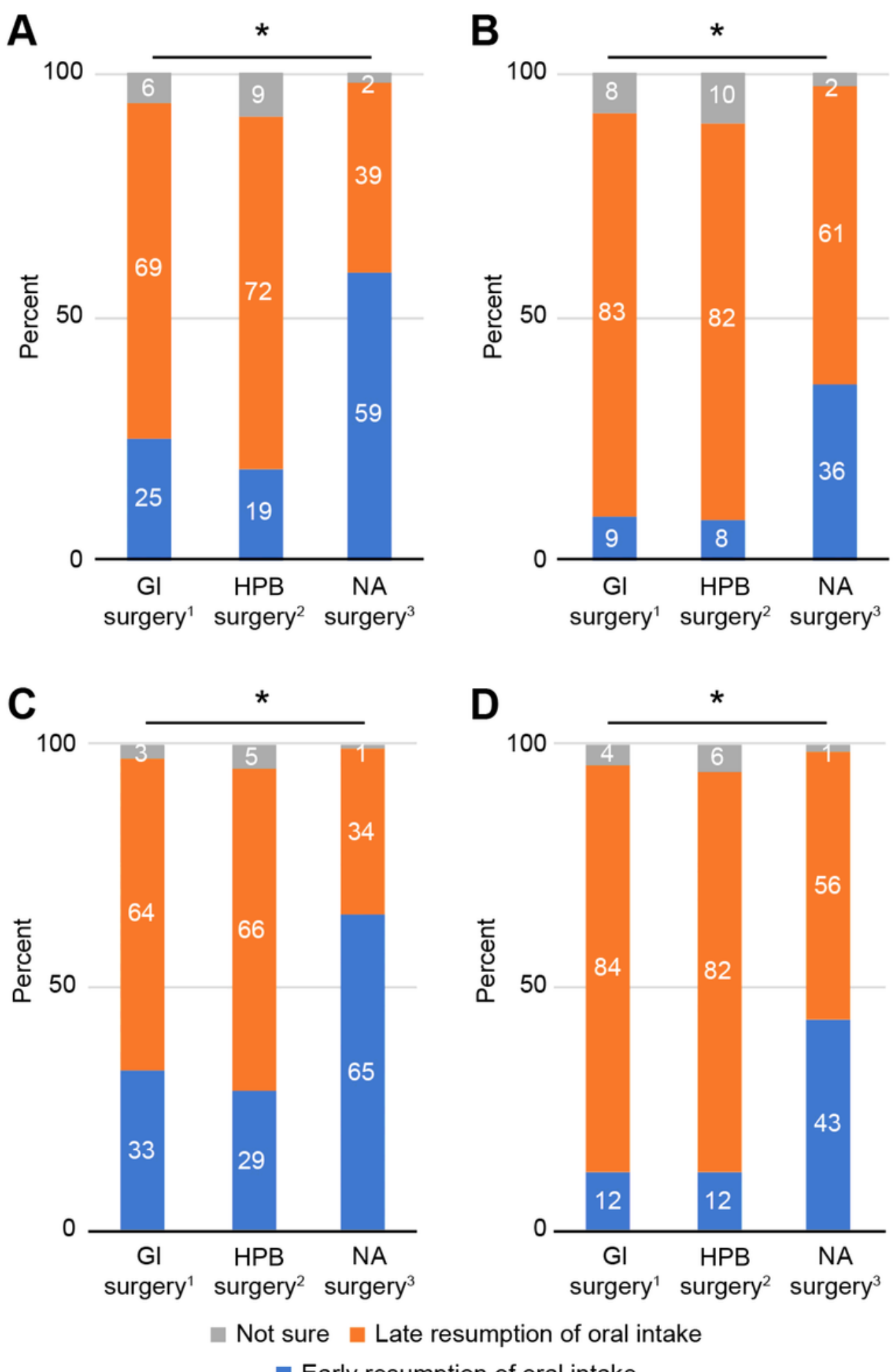

- Early resumption of oral intake

\section{Figure 4}

Divergence among the three surgery types for the postoperative resumption of oral fluids $(A \& C)$ and solid diet (B\& D) in practice (A \& B) and in perception (C \& D). GI surgery, gastrointestinal surgery; HPB surgery, hepato-pancreato-biliary surgery; NA surgery, non-abdominal surgery. Data presented as \% in columns. *,1-6 Wilcoxon signed rank test, $\mathrm{P}<0.001$ 
A

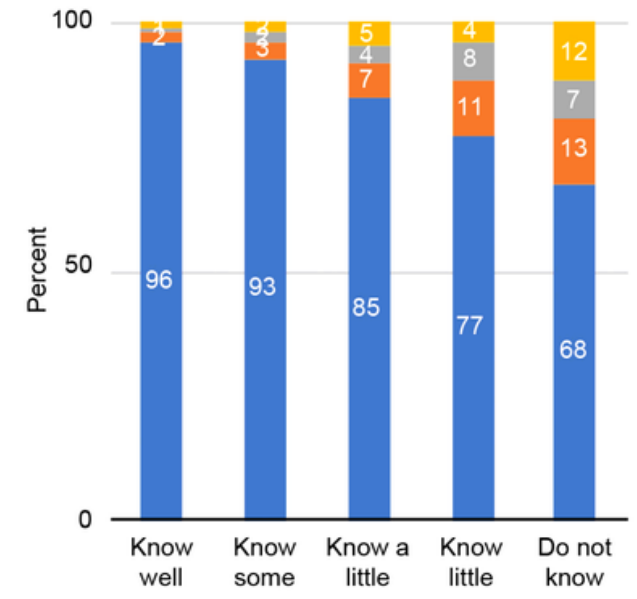

D

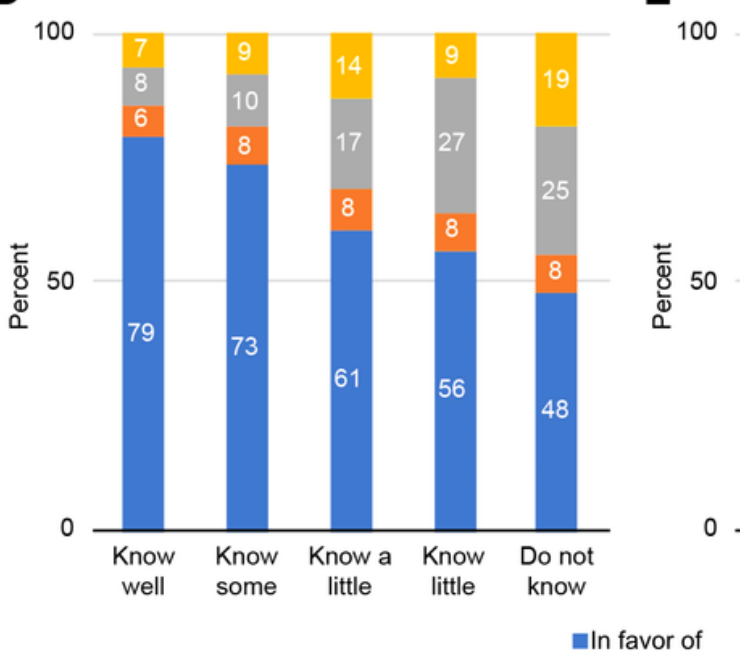

B

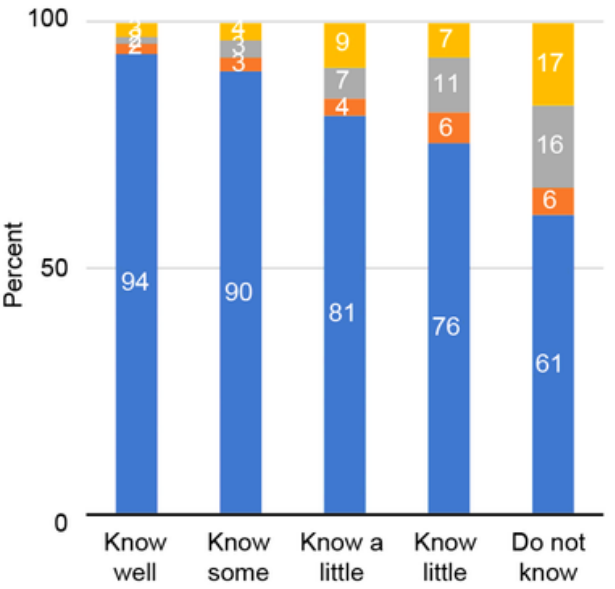

$\mathbf{E}$

100

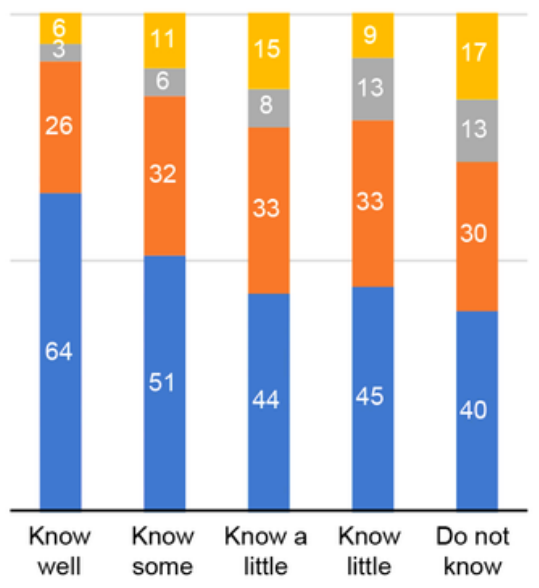

C

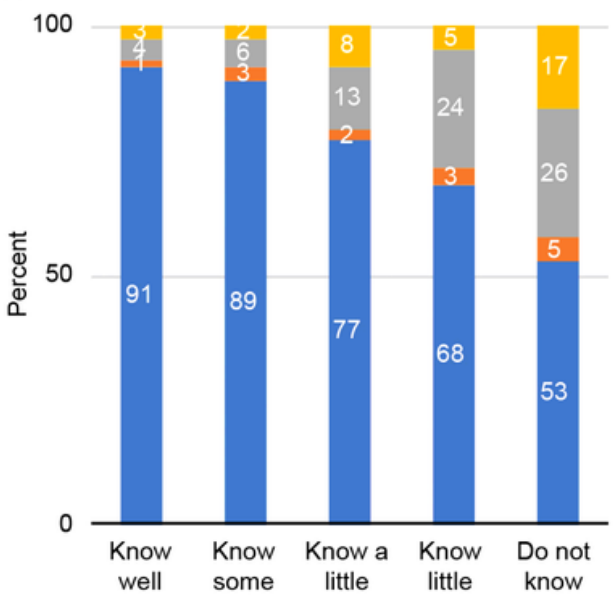

$\mathbf{F}$

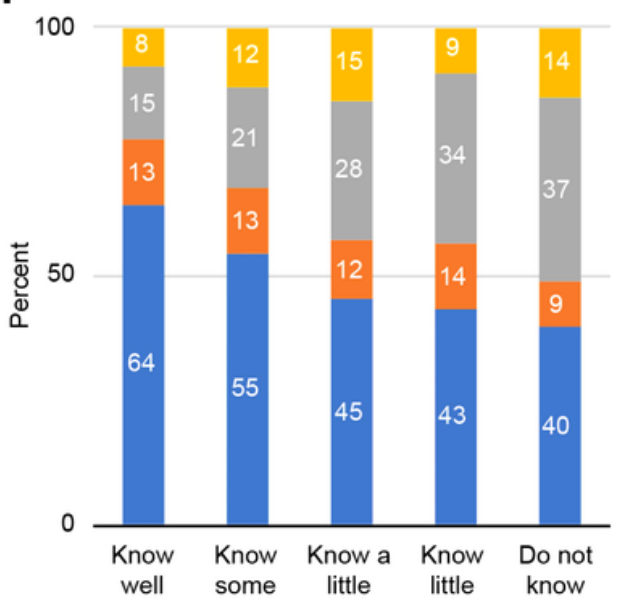

Figure 5

Association of attitudes toward the early resumption of oral intake and understanding of ERAS. (A) Improve patient satisfaction. (B) Improve overall prognosis. (C) Speed up bowel recovery. (D) Reduce postoperative complications. (E) Alleviate postoperative pain. (F) Alleviate postoperative nausea and vomiting. Data presented as \% in columns. 


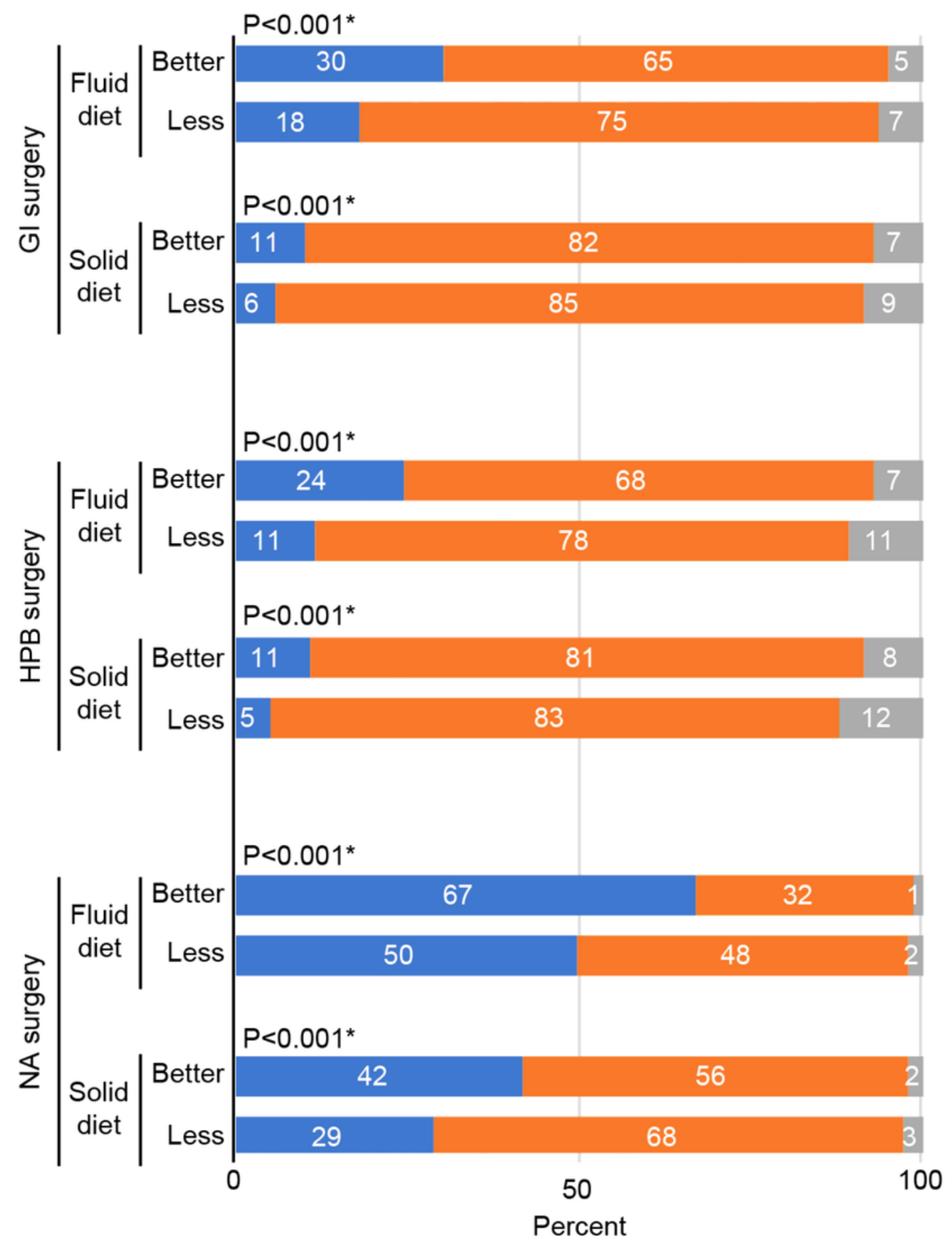

Not sure Late resumption of oral intake

- Early resumption of oral intake

\section{Figure 6}

Understanding of ERAS affected the resumption of oral intake. GI surgery, gastrointestinal surgery; HPB surgery, hepato-pancreato-biliary surgery; NA surgery, non-abdominal surgery; Better = Better understanding of ERAS; Less= Less understanding of ERAS. Data presented as \% in columns. * Chisquare test 


\section{Supplementary Files}

This is a list of supplementary files associated with this preprint. Click to download.

- AppendixA.docx 